\title{
Outreach Activity in Astrobiology: Illusions and Overstatements
}

\section{Ivan Almar*}

Honorary Professor at the Loránd Eötvös, University of Budapest, Department of Astronomy, Hungary

\section{Introduction}

This is a warning to every author involved in outreach activity connected with astrobiology and exoplanets discoveries. I strongly advise: don't use hasty statements or declarations when referring to new results in this field. The problem is that we are accustomed reading or listening to such headlines as "A habitable planet discovered in a far-away planetary system" or "Life-supporting world observed around a Sun-like star" or "Astronomers detected water in the atmosphere of five exoplanets-probably there is life on them" or "Earth-like exoplanets discovered" etc.. These are overstatements for different reasons.

First of all the existence of water, oxygen and ozone might be important life-signatures in the atmosphere of a planet or moon, but nobody have proved that these are necessary and sufficient conditions for life. The only known celestial body, where different life forms exist, is our Earth; extrapolating terrestrial biology to the whole Universe is risky.

Second, we don't know which properties of our Earth are responsible for the origin and survival of terrestrial life. The discovery of extremophiles-even deep in the crust of the Earth, where water and solar irradiation are practically absent-gives some warning that life can adapt itself to very strange conditions even on our planet.

Third, the thousand exoplanets discovered up-till-now belongs to very different categories. Some of them are really Earth-like, because of their mass and diameter-like Venus in our Solar System, which demonstrates that such an Earth-like planet might be totally different in other respects (surface temperature, atmosphere) making it a hell for terrestrial life.

Fourth, even if an exoplanets orbits a normal star in its so called habitable zone there is no reason to believe that it is really inhabited. Our Moon which is situated certainly within the habitable zone of our Sun is a good example.No indigenous life forms have been found there, neither on its surface nor below.

In any case one should be very cautious when using the popular term "habitable zone". Even the exact meaning of this term is not clear at all. As I have formulated in a Billingham Cutting-Edge Lecture in 2008 [1] "My conclusion is that the habitable zone is a completely obsolete term and should not be used at all. In the case of habitability it should be always added in which sense the planet (or other celestial body) is considered habitable."

Namely there are many questions without any satisfactory answer concerning habitability. The classical definition: "habitable=suitable to live in or on" is clearly insufficient. Are celestial bodies habitable only if they dispose of indigenous life? Is it an absolute condition that complex life forms have already developed on their surface? Or is it enough to prove that there is a probability that such life forms may develop there in the future? Or that an alien life form arrived through space may survive there on the surface or under the surface? Or that some terrestrial life is able to adopt itself to the dominant conditions of this alien world? Or a technical civilization might be able to transform these alien surface conditions "habitable" for terrestrial life? and if life does not exist on their surface, but only somewhere inside? Finally is our Moon habitable because a dozen of living astronauts spent some time on it?

It is a popular hypothesis that a habitable celestial body must orbit around a star within its "habitable zone", where any (?) planet can maintain liquid water on its surface. A habitable zone (HZ) is usually defined as the region where a planet could reside and maintain surface temperatures that allow liquid water to exist, i.e. habitability depends only on its distance from the central star. In reality it is well known that in the Solar System surface temperature depends also on the density and composition of the atmosphere of the celestial body (planet or moon), which depends strongly on its mass. Moreover there are several internal heat sources (tides!) which might maintain subsurface oceans even very far from the central star (e.g. Europa).

Consequently nowadays the original concept of habitable zone and habitability underwent essential changes. The title of a recent paper by Sean McMahon is a good example [2]: "Habitable zones around stars ten times wider than we thought." The author argues that "theory fails to take into account life that can exist beneath a planet's surface. As you get deeper ... the temperature increases, and once get down to a temperature where liquid water can exist - life can exist there too." The deepest known life on Earth is $5.3 \mathrm{~km}$ below the surface, but there may well be life even $10 \mathrm{~km}$ deep in places on Earth that have not yet been drilled. In that case - as the author has calculated - Earth-like planets or moons could support life as far as 14 times the distance previously considered to be the "Goldilocks zone" (another popular name of the habitable zone). Moreover his conclusion is that life receives much more protection inside a warm, mineral-rich rock than risking survival on its inhospitable surface, i.e. life on the surface of the Earth could itself be an anomaly.

This kind of definition, however, would allow habitability not only in a special zone within a planetary system, but almost everywhere, even under the surface of a rogue planet or a brown dwarf star. These kind of individual celestial bodies have been recently recognized as important constituents of our Galaxy.

Finally I would like to cite some recent publications to illustrate how bold and risky statements infiltrated into the scientific and semiscientific literature (and consequently into any kind of outreach activity in the media) in this important and interesting field. Hundreds of exciting exoplanets have been discovered during the first years of the exceptionally successful Kepler mission. NASA has announced already in 2011 that "NASA Kepler mission has confirmed its first planet in the

${ }^{*}$ Corresponding author: Ivan Almar, Honorary Professor at the Loránd Eötvös, University of Budapest, Department of Astronomy, Hungary, Tel: 361391 9327, E-mail: almar@konkoly.hu

Received February 05, 2014; Accepted February 06, 2014; Published February 08, 2014

Citation: Almar I (2014) Outreach Activity in Astrobiology: Illusions and Overstatements. Astrobiol Outreach 2: e103. doi: 10.4172/2332-2519.1000e103

Copyright: (c 2014 Almar I. This is an open-access article distributed under the terms of the Creative Commons Attribution License, which permits unrestricted use, distribution, and reproduction in any medium, provided the original author and source are credited. 
habitable zone, the region where liquid water could exist on a planet's surface." The candidate, planet Kepler 22b, has, however, a radius approximately 2.4 times longer than that of our Earth. Nevertheless Kepler scientists declared that "this is a major milestone on the road to finding Earth's twin ... and to detect those that are not only Earth-size but also potentially habitable."

Nowadays authors of the Habitable Exoplanets Catalogue [3] try to rank the habitability of exoplanets using the "Earth Similarity Index" (ESI). ESI is a measure of Earth-likeliness from zero to one (ESI $=1$ is a planet identical to Earth), based on interior parameters (mean radius and bulk density) and surface parameters (escape velocity and surface temperature). The authors of the catalogue are convinced that "only those planets above ESI value 0.8 are suitable for complex life (plants and animals)." In these days Gliese $667 \mathrm{Cc}$ and Kepler 62e are at the top with ESI $=0.83$, but there are no observations confirming that they are really inhabited or even habitable at all.

The last paper to be cited is quite new and introduces the term "superhabitability". Based on model calculations researchers at the Ontario University, Canada, have recently published a paper on Alpha Centauri B [4] claiming that a planet of this neighbor orange star, slightly smaller than our sun, may host a "superhabitable" lifesupporting world (i.e. planet) of islands, shallow seas and gentle slopes. They are convinced that since such a planet of Alpha Centauri B might orbit for 7-10 billion years within the habitable zone of its star, it has "enough time for ecosystems to reach an optimal state for diverse life to flourish".

As a conclusion I repeat that in spite of the remarkable success of exoplanets research in recent years authors should be cautious when declaring discovery of habitable Earth-like exoplanets. Since we don't know the necessary and sufficient conditions of life in the Universe, every such statement needs extraordinary and indisputable observational evidence, otherwise the readers will be misguided.

\section{References}

1. Almar I (2011) SETI terminology: Do we interpret SETI terms correctly? Acta Astronautica 68 : 351-357.

2. McMahon, Sean: Habitable zones around stars ten times wider than we thought, Aberdeen and St. Andrews universities (Space Daily 10.01.2014)

3. Habitable Exoplanets Catalog, University of Puerto Rico.

4. Superhabitable Worlds, University of Ontario. 